\title{
A Beale-Kato-Majda Blow-up Criterion for a Hydrodynamic System Modeling Vesicle and Fluid Interactions*
}

\author{
Jihong Zhao ${ }^{\dagger}$ \\ Institute of Applied Mathematics, College of Science, Northwest A\&F University, \\ Yangling, Shaanxi 712100, People's Republic of China
}

\begin{abstract}
In this paper, we establish an analog of the Beale-Kato-Majda type criterion for singularities of smooth solutions of a hydrodynamic system modeling vesicle and fluid interactions. The result shows that the maximum norm of the vorticity alone controls the breakdown of smooth solutions.

Keywords: Phase field; vesicle membrane; fluid interaction; Navier-Stokes equations; blow-up criterion
\end{abstract}

2010 AMS Subject Classification: 35B65, 35Q30, 76D09, 76T10

\section{Introduction}

During the past several decades, there have been many experimental and mathematical investigations focusing on the formation and dynamics of elastic vesicle membranes $[1,4,5,15,20,22,24,25$. The single component vesicles are elastic membranes containing a liquid and surrounded by another liquid, which are possibly the simplest models for the biological cells and molecules. Such vesicles can be formed by certain amphiphilic molecules assembled in water to build bilayers, and exhibit a rich set of geometric structures in various mechanical, physical and biological environment [10, 21]. Their equilibrium shapes can be characterized by minimizing the following bending elastic energy of the membranes [13]:

$$
E=\int_{\Gamma} \frac{k}{2}\left(H-c_{0}\right)^{2} d S
$$

where $\Gamma$ is the surface of vesicle membrane, $H=\frac{k_{1}+k_{2}}{2}$ is the mean curvature of the membrane surface with $k_{1}$ and $k_{2}$ as the principle curvatures, $c_{0}$ is the spontaneous curvature which arises due to inhomogeneities in the bilayer lipid membrane structure, and $k$ is the bending modulus of the vesicle membrane.

In [6, 10, by using the phase field approach, the authors introduced the phase field NavierStokes vesicle fluid interaction model for the vesicle shape dynamics and conducted numerical

\footnotetext{
*This work is partially supported by the National Natural Science Foundation of China (11171357).

${ }^{\dagger}$ E-mail: zhaojihong2007@yahoo.com.cn.
} 
simulations of the vesicle membrane deformation in flow fields (see [8, 9, 27] for further studies). In this model, the vesicle membrane is described by a phase function $\phi$, which is a labeling function defined on computational domain $Q$. The function $\phi$ takes value +1 inside of the vesicle membrane and -1 outside, with a thin transition layer of width characterized by a small (compared to the vesicle size) positive parameter $\varepsilon$. Obviously, the vesicle membrane $\Gamma$ coincides with the zero level set $\{x: \phi(x)=0\}$. The convergence of the phase field model to the original sharp interface model as the transition width of the diffuse interface $\varepsilon \rightarrow 0$ has been carried out in 7 . On the other hand, the viscous fluid is modeled by the incompressible Navier-Stokes equations with unit density and with an external force defined in terms of $\phi$.

As in [6], for simplicity, we assume that $k$ is a positive constant and $H_{0}=0$. The elastic bending energy (1.1) will be approximated by a modified Willmore energy (cf. [10])

$$
E_{\varepsilon}(\phi)=\frac{k}{2 \varepsilon} \int_{Q}|f(\phi)|^{2} d x \text { with } f(\phi)=-\varepsilon \Delta \phi+\frac{1}{\varepsilon}\left(\phi^{2}-1\right) \phi,
$$

which depends on the interface transitional thickness $\varepsilon$. Moreover, in order to keep the total volume and the surface area of the vesicle membrane are conserved in time, two constraint functionals for the vesicle volume and surface area are prescribed by (cf. [10)

$$
A(\phi)=\int_{Q} \phi d x, \quad B(\phi)=\int_{Q}\left(\frac{\varepsilon}{2}|\nabla \phi|^{2}+\frac{1}{4 \varepsilon}\left(|\phi|^{2}-1\right)^{2}\right) d x
$$

To enforce these constraints, two penalty terms were added to the elastic bending energy $E_{\varepsilon}(\phi)$, and the approximate elastic bending energy is given by (cf. [11, 12])

$$
E(\phi)=E_{\varepsilon}(\phi)+\frac{1}{2} M_{1}(A(\phi)-\alpha)^{2}+\frac{1}{2} M_{2}(B(\phi)-\beta)^{2},
$$

where $M_{1}$ and $M_{2}$ are two penalty constants, $\alpha=A\left(\phi_{0}\right)$ and $\beta=B\left(\phi_{0}\right)$ are determined by the initial value of the phase function $\phi_{0}$.

In this paper, we consider the three dimensional phase field Navier-Stokes vesicle fluid interaction model subjecting to the periodic boundary conditions (i.e., in torus $\mathbb{T}^{3}$ ), which reads as follows:

$$
\begin{array}{ll}
\partial_{t} u+u \cdot \nabla u+\nabla P=\mu \Delta u+\frac{\delta E(\phi)}{\delta \phi} \nabla \phi & \text { in } Q \times[0, T], \\
\nabla \cdot u=0 & \text { in } Q \times[0, T], \\
\partial_{t} \phi+u \cdot \nabla \phi=-\gamma \frac{\delta E(\phi)}{\delta \phi} & \text { in } Q \times[0, T]
\end{array}
$$

with the initial condition

$$
u(x, 0)=u_{0}(x) \text { with } \nabla \cdot u_{0}=0 \text { and } \phi(x, 0)=\phi_{0}(x) \text { for } x \in Q,
$$

and the boundary condition

$$
u\left(x+e_{i}, t\right)=u(x, t), \quad \phi\left(x+e_{i}, t\right)=\phi(x, t) \text { for } x \in \partial Q \times[0, T],
$$


where the set of vectors $\left\{e_{1}=(1,0,0), e_{2}=(0,1,0), e_{3}=(0,0,1)\right\}$ denotes an orthonormal basis of $\mathbb{R}^{3}$ and $Q$ is the unit square in $\mathbb{R}^{3}$. Here $u=\left(u_{1}, u_{2}, u_{3}\right) \in \mathbb{R}^{3}$ and $P \in \mathbb{R}$ denote, respectively, the velocity field and the pressure of the fluid, $\phi \in \mathbb{R}$ is the phase function of the vesicle membrane. $\frac{\delta E(\phi)}{\delta \phi}$ is the so-called chemical potential that denotes the variational derivative of $E(\phi)$ in the variable $\phi . \quad \gamma$ denotes the mobility coefficient which is assumed to be a small positive constant. Note that, if we denote

$$
g(\phi)=-\Delta f(\phi)+\frac{1}{\varepsilon^{2}}\left(3 \phi^{2}-1\right) f(\phi)
$$

then

$$
\begin{aligned}
\frac{\delta E(\phi)}{\delta \phi}= & k g(\phi)+M_{1}(A(\phi)-\alpha)+M_{2}(B(\phi)-\beta) f(\phi) \\
= & k \varepsilon \Delta^{2} \phi-\frac{k}{\varepsilon} \Delta\left(\phi^{3}-\phi\right)-\frac{k}{\varepsilon}\left(3 \phi^{2}-1\right) \Delta \phi+\frac{k}{\varepsilon^{3}}\left(3 \phi^{2}-1\right)\left(\phi^{2}-1\right) \phi \\
& +M_{1}(A(\phi)-\alpha)+M_{2}(B(\phi)-\beta) f(\phi) .
\end{aligned}
$$

The system (1.5)-(1.7) describes the evolution of vesicle membranes immersed in an incompressible viscous fluid. Equations (1.5) and (1.6) are the momentum conservation and the mass conservation equations of a viscous fluid with unit density and with an external force caused by the phase field $\phi$. Equation (1.6) is the condition of incompressibility. Equation (1.7) is a relaxed transport equation of $\phi$ with advection by the velocity field $u$. The right-hand side of (1.7) is a regularization term which ensures the consistent dissipation of energy. Roughly speaking, the system (1.5)-(1.7) is governed by the coupling of the hydrodynamic fluid flow and the bending elastic properties of the vesicle membrane. The resulting membrane configuration and the flow field reflect the competition and the coupling of the kinetic energy and membrane elastic energies.

Based on the following basic energy law:

$$
\frac{d}{d t}\left(\frac{1}{2}\|u(\cdot, t)\|_{L^{2}}^{2}+E(\phi(\cdot, t))\right)+\mu\|\nabla u(\cdot, t)\|_{L^{2}}^{2}+\gamma\left\|\frac{\delta E(\phi)}{\delta \phi}\right\|_{L^{2}}^{2}=0, \quad \forall t>0,
$$

the global existence of weak solution to the system (1.5)-(1.7) subject to no-slip boundary condition for the velocity field and Dirichlet boundary condition for the phase function has been established in [6] via the Galerkin argument. Moreover, the authors also proved the weak solution is unique under an additionally regularity assumption $u \in L^{8}\left(0, T ; L^{4}(Q)\right)$. Recently, local in time existence and uniqueness of strong solution to the system (1.5) (1.7) have been established in 19, and under the assumption that the initial data and the quantity $(|\Omega|+\alpha)^{2}$ are sufficiently small, the authors proved existence of almost global strong solutions. Note that they have to restrict the working space with proper limited regularity due to some compatibility conditions at the boundary which is required in the fixed point strategy. Very recently, $\mathrm{Wu}$ and $\mathrm{Xu}[28$, considered the system (1.5)-(1.7) with periodic boundary conditions to avoid troubles caused by the boundary terms when performing integration by parts. They proved that, for any given initial data $\left(u_{0}, \phi_{0}\right) \in H_{p e r}^{1}(Q) \times H_{p e r}^{4}(Q)$, there exists a positive time $T$ such that the system (1.5)-(1.9) admits a unique smooth solution $(u, \phi)$ satisfying

$$
\left\{\begin{array}{l}
u \in C\left([0, T], H_{p e r}^{1}(Q)\right) \cap L^{2}\left(0, T ; H_{p e r}^{2}(Q)\right) \cap H^{1}\left(0, T ; L_{p e r}^{2}(Q)\right), \\
\phi \in C\left([0, T], H_{p e r}^{4}(Q)\right) \cap L^{2}\left(0, T ; H_{p e r}^{6}(Q)\right) \cap H^{1}\left(0, T ; H_{p e r}^{2}(Q)\right) .
\end{array}\right.
$$


Moreover, if the viscosity $\mu$ is assumed to be properly large, then the system (1.5)-(1.9) admits a unique global strong solution that is uniformly bounded in $H_{p e r}^{1} \times H_{p e r}^{4}$ on $[0, \infty)$. However, as for the well-known Navier-Stokes equations, an outstanding open problem is whether or not smooth solution of (1.5) -(1.9) on $[0, T)$ will lead to a singularity at $t=T$. For this purpose, they established some regularity criteria for local smooth solutions in terms of the velocity field only. More precisely, they proved that if one of the following conditions holds:

$$
\begin{aligned}
& \text { (i) } \int_{0}^{T}\|u(\cdot, t)\|_{L^{r}}^{q} d t<\infty \quad \text { with } \frac{3}{r}+\frac{2}{q} \leq 1,3<r \leq \infty, \\
& \text { (ii) } \int_{0}^{T}\|\nabla u(\cdot, t)\|_{L^{r}}^{q} d t<\infty \text { with } \frac{3}{r}+\frac{2}{q} \leq 2, \frac{3}{2}<r \leq \infty,
\end{aligned}
$$

then the solution $(u, \phi)$ can be extended beyond the time $T$. Some improved logarithmical-type regularity criteria as for the conventional Navier-Stokes equations were also established in [28], and the stability of the system (1.5)-(1.9) near local minimizers of the elastic bending energy were obtained by using the Eojasiewicz-Simon inequality.

For the Navier-Stokes equations, the regularity criteria (1.14) and (1.15) were obtained by Prodi [23], Serrin [26], Giga [14] and Beirão da Veiga [3]. In order to replace $\nabla u$ by the vorticity $\nabla \times u$ in the condition (1.15), the case $1<r<\infty$ is a simple case because the Biot-Savart law and the boundness of the Riesz transforms on $L^{r}$. However, the marginal case $r=\infty$ is very difficult due to the lack of continuity of Riesz transforms on $L^{\infty}$. In 1984, Beale-Kato-Majda in their pioneering work 2] got around this difficulty by using the logarithmic Sobolev inequality and showed that if the smooth solution $u$ blows up at the time $t=T$, then

$$
\int_{0}^{T}\|\omega(\cdot, t)\|_{L^{\infty}} d t=\infty
$$

where $\omega=\nabla \times u$ is the vorticity of the velocity field. Later, Kozono-Taniuchi [18] and KonozoOgawa-Taniuchi [17] refined (1.16) to

$$
\int_{0}^{T}\|\omega(\cdot, t)\|_{B M O} d t=\infty \text { and } \int_{0}^{T}\|\omega(\cdot, t)\|_{\dot{B}_{\infty, \infty}^{0}} d t=\infty
$$

respectively, where $B M O$ is the space of Bounded Mean Oscillation and $\dot{B}_{\infty, \infty}^{0}$ is the homogeneous Besov spaces.

Inspired by 2] and [28, the purpose of this paper is to establish an analog of Beale-Kato-Majda's criterion for singularities of smooth solutions to the system (1.5)-(1.9). Our result shows that the maximum norm of the vorticity alone controls the breakdown of smooth solutions, and more important, it reveals that the velocity field $u$ plays a more dominant role than the phase function $\phi$ in the regularity theory of solutions to the phase field Navier-Stokes vesicle-fluid interaction system (1.5) -(1.9). As in 22], the proof will be accomplished in three steps: obtaining $L^{2}$ estimates for the vorticity $\omega$ and $\nabla \Delta \phi$, obtaining higher energy estimates for the solution $(u, \phi)$, and applying the crucial logarithmic Sobolev inequality.

Before stating our main result, we recall some well-established functional settings for periodic problems: For $1 \leq r \leq \infty$, we denote by

$$
L_{p e r}^{r}(Q):=\left\{u \in L^{r}\left(\mathbb{R}^{3}\right) \mid u\left(x+e_{i}\right)=u(x)\right\}
$$


equipped the usual norm

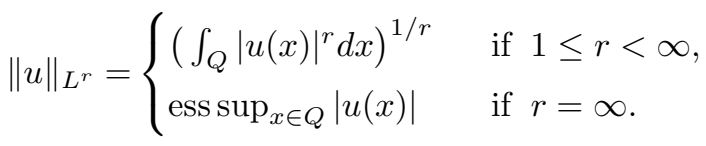

For an interger $m>0$, we denote by

$$
H_{p e r}^{m}(Q):=\left\{u \in H^{m}\left(\mathbb{R}^{3}\right) \mid u\left(x+e_{i}\right)=u(x)\right\}
$$

equipped with the usual norm $\|u\|_{H^{m}}=\sum_{i=0}^{m}\left\|\nabla^{i} u\right\|_{L^{2}}$.

Our main result reads as follows:

Theorem 1.1 Assume that $\left(u_{0}, \phi_{0}\right) \in H_{\text {per }}^{3}(Q) \times H_{\text {per }}^{6}(Q)$ with $\nabla \cdot u_{0}=0$. Let $T_{*}>0$ be the maximum existence time such that the system (1.5)-(1.9) has a unique strong solution $(u, \phi)$ on $\left[0, T_{*}\right)$. If $T_{*}<\infty$, then

$$
\int_{0}^{T_{*}}\|\omega(\cdot, t)\|_{L^{\infty}} d t=\infty
$$

In particular,

$$
\limsup _{t \nearrow T_{*}}\|\omega(\cdot, t)\|_{L^{\infty}}=\infty .
$$

The proof of Theorem 1.1 yields the following immediate consequence:

Corollary 1.2 Assume that $\left(u_{0}, \phi_{0}\right) \in H_{\text {per }}^{3}(Q) \times H_{\text {per }}^{6}(Q)$ with $\nabla \cdot u_{0}=0$. Let $(u, \phi)$ be the corresponding local strong solution to the system (1.5)-(1.9) on $[0, T)$ for some $0<T<\infty$. If

$$
\int_{0}^{T}\|\omega(\cdot, t)\|_{L^{\infty}} d t<\infty
$$

then the solution $(u, \phi)$ can be extended past time $t=T$.

In the next section, we give the proof of Theorem 1.1 .

\section{The proof of Theorem 1.1}

We prove Theorem 1.1 by contradiction. Since $\left(u_{0}, \phi_{0}\right) \in H_{p e r}^{3}(Q) \times H_{p e r}^{6}(Q)$ with $\nabla \cdot u_{0}=0$, we deduce from [28] that there exists $0<T<\infty$ such that the problem (1.5)-(1.9) admits a unique strong solution $(u, \phi)$ satisfying

$$
\left\{\begin{array}{l}
u \in C\left([0, T], H_{p e r}^{3}(Q)\right) \cap L^{2}\left(0, T ; H_{p e r}^{4}(Q)\right) \cap H^{1}\left(0, T ; H_{p e r}^{2}(Q)\right), \\
\phi \in C\left([0, T], H_{p e r}^{6}(Q)\right) \cap L^{2}\left(0, T ; H_{p e r}^{8}(Q)\right) \cap H^{1}\left(0, T ; H_{p e r}^{4}(Q)\right) .
\end{array}\right.
$$

Moreover, if $(u, \phi)$ does not belong to the class (2.1) then it must be that

$$
\limsup _{t \nearrow T}\left(\|u(\cdot, t)\|_{H^{3}}+\|\phi(\cdot, t)\|_{H^{6}}\right)=\infty .
$$


In fact, if this is not true, then there exists $C_{0}>0$ such that $\|u(\cdot, t)\|_{H^{3}}+\|\phi(\cdot, t)\|_{H^{6}} \leq C_{0}$ for all $t<T$. By the local existence theorem stated in [28, we can start a solution at any time $t_{1}$ with initial value $\left(u\left(x, t_{1}\right), \phi\left(x, t_{1}\right)\right)$, and this solution will be regular for $t_{1}<t<t_{1}+T_{0}\left(C_{0}\right)$, with $T_{0}$ independent of $t_{1}$. By choosing $t_{1} \in\left(T-T_{0}, T\right)$, we have then extended the original solution past to the time $T$, contrary to the choice of $\mathrm{T}$.

Therefore, it suffices to show that if the condition (1.18) holds, then

$$
\|u(\cdot, t)\|_{H^{3}}+\|\phi(\cdot, t)\|_{H^{6}} \leq C, \quad 0 \leq t \leq T
$$

for some constant $C$ depending only on $T,\left\|u_{0}\right\|_{H^{3}},\left\|\phi_{0}\right\|_{H^{6}}$, coefficients of the system (1.5)-(1.9), and

$$
K:=\int_{0}^{T}\|\omega(\cdot, t)\|_{L^{\infty}} d t<\infty
$$

Let us first establish $L^{2}$ estimate of the vorticity $\omega$ and $\nabla \Delta \phi$. Recall that we have already obtained the following uniform estimates (cf. [6, 28]):

$$
\left\{\begin{array}{l}
\|u(\cdot, t)\|_{L^{2}}+\|\phi(\cdot, t)\|_{H^{2}} \leq C \text { for all } t \geq 0 \\
\int_{0}^{+\infty}\left(\mu\|\nabla u(\cdot, t)\|_{L^{2}}^{2}+\gamma\left\|\frac{\delta E}{\delta \phi}(\cdot, t)\right\|_{L^{2}}^{2}\right) d t \leq C
\end{array}\right.
$$

where $C$ is a constant depending only on $\left\|u_{0}\right\|_{L^{2}},\left\|\phi_{0}\right\|_{H^{2}}$ and coefficients of the system except the viscosity $\mu$.

Lemma 2.1 Assume that $\left(u_{0}, \phi_{0}\right) \in H_{p e r}^{3}(Q) \times H_{p e r}^{6}(Q)$ with $\nabla \cdot u_{0}=0$. Let $(u, \phi)$ be the corresponding local smooth solution to the system (1.5)-(1.9) on [0,T) for some $0<T<\infty$. If

$$
\int_{0}^{T}\|\omega(\cdot, t)\|_{L^{\infty}} d t=K<\infty
$$

then

$$
\sup _{0 \leq t \leq T}\left(\|\omega(\cdot, t)\|_{L^{2}}^{2}+\|\nabla \Delta \phi(\cdot, t)\|_{L^{2}}^{2}\right) \leq C
$$

where $C$ is a constant depending only on $\left\|u_{0}\right\|_{H^{1}},\left\|\phi_{0}\right\|_{H^{3}}, K$ and coefficients of the system.

Proof. Taking the curl on (1.5), we obtain

$$
\partial_{t} \omega-\mu \Delta \omega+u \cdot \nabla \omega=\omega \cdot \nabla u+\nabla \times\left(\frac{\delta E}{\delta \phi} \nabla \phi\right) .
$$

Multiplying (2.6) by $\omega$ and integrating over $Q$,

$$
\frac{1}{2} \frac{d}{d t}\|\omega\|_{L^{2}}^{2}+\mu\|\nabla \omega\|_{L^{2}}^{2}=\int_{Q} w \cdot \nabla u \cdot \omega d x-\int_{Q} \frac{\delta E}{\delta \phi} \nabla \phi \cdot \nabla \times \omega d x
$$

where we have used the fact $\int_{Q} u \cdot \nabla \omega \cdot \omega d x=0$ due to $\nabla \cdot u=0$. Since the Riesz operators are bounded in $L^{2}$ and $\nabla u=(-\Delta)^{-1} \nabla(\nabla \times \omega)$, we have $\|\nabla u\|_{L^{2}} \leq C\|\omega\|_{L^{2}}$. This implies that

$$
\left|\int_{Q} w \cdot \nabla u \cdot \omega d x\right| \leq C\|\omega\|_{L^{\infty}}\|\nabla u\|_{L^{2}}\|\omega\|_{L^{2}} \leq C\|\omega\|_{L^{\infty}}\|\omega\|_{L^{2}}^{2}
$$


Applying Young's inequality and (2.3), we have

$$
\begin{aligned}
\left|\int_{Q} \frac{\delta E}{\delta \phi} \nabla \phi \cdot \nabla \times \omega d x\right| & \leq \frac{\mu}{2}\|\nabla \omega\|_{L^{2}}^{2}+C\left\|\frac{\delta E}{\delta \phi} \nabla \phi\right\|_{L^{2}}^{2} \\
& \leq \frac{\mu}{2}\|\nabla \omega\|_{L^{2}}^{2}+C\left\|\frac{\delta E}{\delta \phi}\right\|_{L^{2}}^{2}\|\nabla \phi\|_{L^{\infty}}^{2} \\
& \leq \frac{\mu}{2}\|\nabla \omega\|_{L^{2}}^{2}+C\left\|\frac{\delta E}{\delta \phi}\right\|_{L^{2}}^{2}\left(\|\nabla \Delta \phi\|_{L^{2}}^{2}+\|\nabla \phi\|_{L^{2}}^{2}\right) \\
& \leq \frac{\mu}{2}\|\nabla \omega\|_{L^{2}}^{2}+C\left\|\frac{\delta E}{\delta \phi}\right\|_{L^{2}}^{2}\left(\|\nabla \Delta \phi\|_{L^{2}}^{2}+1\right),
\end{aligned}
$$

where we have used the Sobolev embedding $H^{2}(Q) \hookrightarrow L^{\infty}(Q)$ and $\|\nabla \phi\|_{L^{\infty}} \leq C\left(\|\nabla \Delta \phi\|_{L^{2}}+\right.$ $\|\nabla \phi\|_{L^{2}}$ ). Taking (2.8) and (2.9) into (2.7), we obtain

$$
\frac{d}{d t}\|\omega\|_{L^{2}}^{2}+\mu\|\nabla \omega\|_{L^{2}}^{2} \leq C\left(\|\omega\|_{L^{\infty}}+\left\|\frac{\delta E}{\delta \phi}\right\|_{L^{2}}^{2}\right)\left(\|\omega\|_{L^{2}}^{2}+\|\nabla \Delta \phi\|_{L^{2}}^{2}+1\right) .
$$

Taking $\Delta$ on (1.7), multiplying the resultant by $-\Delta^{2} \phi$, and integrating over $Q$, we obtain

$$
\frac{1}{2} \frac{d}{d t}\|\nabla \Delta \phi\|_{L^{2}}^{2}=-\int_{Q} \nabla \cdot(u \cdot \nabla \phi) \cdot \nabla \Delta^{2} \phi d x-\gamma \int_{Q} \nabla \frac{\delta E}{\delta \phi} \cdot \nabla \Delta^{2} \phi d x:=I_{1}+I_{2} .
$$

By (2.3), $I_{1}$ can be directly estimated as follows:

$$
\begin{aligned}
I_{1} & \leq \frac{k \gamma \varepsilon}{8}\left\|\nabla \Delta^{2} \phi\right\|_{L^{2}}^{2}+C\|\nabla u \cdot \nabla \phi\|_{L^{2}}^{2}+C\left\|u \cdot \nabla^{2} \phi\right\|_{L^{2}}^{2} \\
& \leq \frac{k \gamma \varepsilon}{8}\left\|\nabla \Delta^{2} \phi\right\|_{L^{2}}^{2}+C\|\nabla u\|_{L^{2}}^{2}\|\nabla \phi\|_{L^{\infty}}^{2}+C\|u\|_{L^{6}}^{2}\left\|\nabla^{2} \phi\right\|_{L^{3}}^{2} \\
& \leq \frac{k \gamma \varepsilon}{8}\left\|\nabla \Delta^{2} \phi\right\|_{L^{2}}^{2}+C\|\nabla u\|_{L^{2}}^{2}\left(\|\nabla \Delta \phi\|_{L^{2}}^{2}+1\right)+C\|\nabla u\|_{L^{2}}^{2}\left\|\nabla^{2} \phi\right\|_{L^{2}}\|\nabla \Delta \phi\|_{L^{2}} \\
& \leq \frac{k \gamma \varepsilon}{8}\left\|\nabla \Delta^{2} \phi\right\|_{L^{2}}^{2}+C\|\nabla u\|_{L^{2}}^{2}\left(\|\nabla \Delta \phi\|_{L^{2}}^{2}+1\right),
\end{aligned}
$$

where we have used the Sobolev embedding $H^{1}(Q) \hookrightarrow L^{6}(Q)$ and the interpolation inequality $\left\|\nabla^{2} \phi\right\|_{L^{3}}^{2} \leq C\left\|\nabla^{2} \phi\right\|_{L^{2}}\|\nabla \Delta \phi\|_{L^{2}}$. For $I_{2}$, since $A(\phi)$ and $B(\phi)$ are functions depending only on time, by (1.10) and (1.11), we have

$$
\begin{aligned}
I_{2}= & -\gamma \int_{Q} \nabla\left[k g(\phi)+M_{1}(A(\phi)-\alpha)+M_{2}(B(\phi)-\beta) f(\phi)\right] \cdot \nabla \Delta^{2} \phi d x \\
= & k \gamma \int_{Q} \nabla \Delta f(\phi) \cdot \nabla \Delta^{2} \phi d x-\frac{k \gamma}{\varepsilon^{2}} \int_{Q} \nabla\left[\left(3 \phi^{2}-1\right) f(\phi)\right] \cdot \nabla \Delta^{2} \phi d x \\
& -M_{2} \gamma(B(\phi)-\beta) \int_{Q} \nabla f(\phi) \cdot \nabla \Delta^{2} \phi d x \\
:= & I_{21}+I_{22}+I_{23} .
\end{aligned}
$$

Note that $f(\phi)=-\varepsilon \Delta \phi+\frac{1}{\varepsilon}\left(\phi^{2}-1\right) \phi$, by (2.3) , we obtain

$$
I_{21}=-k \varepsilon \gamma\left\|\nabla \Delta^{2} \phi\right\|_{L^{2}}^{2}+\frac{k \gamma}{\varepsilon} \int_{Q} \nabla \Delta\left(\phi^{3}-\phi\right) \cdot \nabla \Delta^{2} \phi d x
$$




$$
\begin{aligned}
& \leq-\frac{7 k \varepsilon \gamma}{8}\left\|\nabla \Delta^{2} \phi\right\|_{L^{2}}^{2}+C\left\|\nabla \Delta\left(\phi^{3}-\phi\right)\right\|_{L^{2}}^{2} \\
& \leq-\frac{7 k \varepsilon \gamma}{8}\left\|\nabla \Delta^{2} \phi\right\|_{L^{2}}^{2}+C\left(\|\phi\|_{L^{\infty}}^{4}\|\nabla \Delta \phi\|_{L^{2}}^{2}+\|\phi\|_{L^{\infty}}^{2}\|\nabla \phi\|_{L^{6}}^{2}\|\Delta \phi\|_{L^{3}}^{2}\right. \\
& \left.+\|\nabla \phi\|_{L^{6}}^{6}+\|\nabla \Delta \phi\|_{L^{2}}^{2}\right) \\
& \leq-\frac{7 k \varepsilon \gamma}{8}\left\|\nabla \Delta^{2} \phi\right\|_{L^{2}}^{2}+C\left(\|\nabla \Delta \phi\|_{L^{2}}^{2}+1\right) \\
& I_{22}=-\frac{6 k \gamma}{\varepsilon^{2}} \int_{Q} \phi \nabla \phi f(\phi) \cdot \nabla \Delta^{2} \phi d x-\frac{k \gamma}{\varepsilon^{2}} \int_{Q}\left(3 \phi^{2}-1\right) \nabla f(\phi) \cdot \nabla \Delta^{2} \phi d x \\
& \leq \frac{k \varepsilon \gamma}{8}\left\|\nabla \Delta^{2} \phi\right\|_{L^{2}}^{2}+C\left(\|\phi \nabla \phi f(\phi)\|_{L^{2}}^{2}+\left\|\left(3 \phi^{2}-1\right) \nabla f(\phi)\right\|_{L^{2}}^{2}\right) \\
& \leq \frac{k \varepsilon \gamma}{8}\left\|\nabla \Delta^{2} \phi\right\|_{L^{2}}^{2}+C\left(\|\phi \nabla \phi \Delta \phi\|_{L^{2}}^{2}+\left\|\phi^{2}\left(\phi^{2}-1\right) \nabla \phi\right\|_{L^{2}}^{2}\right. \\
& \left.+\left\|\left(3 \phi^{2}-1\right) \nabla \Delta \phi\right\|_{L^{2}}^{2}+\left\|\left(3 \phi^{2}-1\right) \nabla\left(\phi^{3}-\phi\right)\right\|_{L^{2}}^{2}\right) \\
& \leq \frac{k \varepsilon \gamma}{8}\left\|\nabla \Delta^{2} \phi\right\|_{L^{2}}^{2}+C\left(\|\phi\|_{L^{\infty}}^{2}\|\nabla \phi\|_{L^{6}}^{2}\|\Delta \phi\|_{L^{3}}^{2}+\|\phi\|_{L^{\infty}}^{4}\left\|\phi^{2}-1\right\|_{L^{\infty}}^{2}\|\nabla \phi\|_{L^{2}}^{2}\right. \\
& \left.+\left\|3 \phi^{2}-1\right\|_{L^{\infty}}^{2}\|\nabla \Delta \phi\|_{L^{2}}^{2}+\left\|3 \phi^{2}-1\right\|_{L^{\infty}}^{4}\|\nabla \phi\|_{L^{\infty}}^{2}\right) \\
& \leq \frac{k \varepsilon \gamma}{8}\left\|\nabla \Delta^{2} \phi\right\|_{L^{2}}^{2}+C\left(\|\nabla \Delta \phi\|_{L^{2}}^{2}+1\right) \\
& I_{23}=M_{2} \varepsilon \gamma(B(\phi)-\beta) \int_{Q} \nabla \Delta \phi \cdot \nabla \Delta^{2} \phi d x-\frac{M_{2} \gamma(B(\phi)-\beta)}{\varepsilon} \int_{Q} \nabla\left(\phi^{3}-\phi\right) \cdot \nabla \Delta^{2} \phi d x \\
& \leq \frac{k \varepsilon \gamma}{8}\left\|\nabla \Delta^{2} \phi\right\|_{L^{2}}^{2}+C(B(\phi)-\beta)^{2}\left(\|\nabla \Delta \phi\|_{L^{2}}^{2}+\left\|\nabla\left(\phi^{3}-\phi\right)\right\|_{L^{2}}^{2}\right) \\
& \leq \frac{k \varepsilon \gamma}{8}\left\|\nabla \Delta^{2} \phi\right\|_{L^{2}}^{2}+C\left(\|\nabla \phi\|_{L^{2}}^{4}+\left\|\phi^{2}-1\right\|_{L^{2}}^{4}+1\right)\left(\|\nabla \Delta \phi\|_{L^{2}}^{2}+\left\|\phi^{2}-1\right\|_{L^{\infty}}^{2}\|\nabla \phi\|_{L^{2}}^{2}\right) \\
& \leq \frac{k \varepsilon \gamma}{8}\left\|\nabla \Delta^{2} \phi\right\|_{L^{2}}^{2}+C\left(\|\nabla \Delta \phi\|_{L^{2}}^{2}+1\right) .
\end{aligned}
$$

Combining the above estimates (2.11)-(2.16), we obtain

$$
\frac{d}{d t}\|\nabla \Delta \phi\|_{L^{2}}^{2}+k \varepsilon \gamma\left\|\nabla \Delta^{2} \phi\right\|_{L^{2}}^{2} \leq C\left(\|\nabla u\|_{L^{2}}^{2}+1\right)\left(\|\nabla \Delta \phi\|_{L^{2}}^{2}+1\right) .
$$

Putting (2.10) and (2.17) together yield that

$$
\begin{aligned}
\frac{d}{d t}\left(\|\omega\|_{L^{2}}^{2}\right. & \left.+\|\nabla \Delta \phi\|_{L^{2}}^{2}\right)+\mu\|\nabla \omega\|_{L^{2}}^{2}+k \varepsilon \gamma\left\|\nabla \Delta^{2} \phi\right\|_{L^{2}}^{2} \\
& \leq C\left(\|\omega\|_{L^{\infty}}+\|\nabla u\|_{L^{2}}^{2}+\left\|\frac{\delta E}{\delta \phi}\right\|_{L^{2}}^{2}+1\right)\left(\|\omega\|_{L^{2}}^{2}+\|\nabla \Delta \phi\|_{L^{2}}^{2}+1\right) .
\end{aligned}
$$

Applying Gronwall's inequality, we conclude that

$$
\|\omega(t)\|_{L^{2}}^{2}+\|\nabla \Delta \phi(t)\|_{L^{2}}^{2} \leq C_{0} \exp \left\{C \int_{0}^{t}\left(\|\omega(s)\|_{L^{\infty}}+\|\nabla u(s)\|_{L^{2}}^{2}+\left\|\frac{\delta E}{\delta \phi}(s)\right\|_{L^{2}}^{2}+1\right) d s\right\},
$$

where $C_{0}=\left(\left\|\omega_{0}\right\|_{L^{2}}^{2}+\left\|\nabla \Delta \phi_{0}\right\|_{L^{2}}^{2}\right)$. By (2.3) and (2.4), we get (2.5). The proof of Lemma 2.1] is complete. 
Combining (2.3) and (2.5), we can easily see that

$$
\sup _{0 \leq t \leq T}\|\phi(\cdot, t)\|_{H^{3}} \leq C,
$$

where $C$ is a constant depending only on $\left\|u_{0}\right\|_{H^{1}},\left\|\phi_{0}\right\|_{H^{3}}, K$ and coefficients of the system. By the Sobolev embedding $H^{2}(Q) \hookrightarrow L^{\infty}(Q)$, (2.20) implies that

$$
\sup _{0 \leq t \leq T}\|\nabla \phi(\cdot, t)\|_{L^{\infty}} \leq C .
$$

This result will be used frequently in the proof of Theorem 1.1

Next, let us derive higher order energy estimates of the solution $(u, \phi)$. Taking $\nabla \Delta$ on (1.5), multiplying the resultant with $\nabla \Delta u$ and integrating over $Q$, we obtain

$$
\begin{aligned}
& \frac{1}{2} \frac{d}{d t}\|\nabla \Delta u\|_{L^{2}}^{2}+\mu\left\|\Delta^{2} u\right\|_{L^{2}}^{2}=-\int_{Q} \nabla \Delta(u \cdot \nabla u) \cdot \nabla \Delta u d x+\int_{Q} \nabla \Delta\left(\frac{\delta E}{\delta \phi} \nabla \phi\right) \cdot \nabla \Delta u d x \\
&:=J_{1}+J_{2} .
\end{aligned}
$$

Since $\nabla \cdot u=0, J_{1}$ can be rewritten as

$$
J_{1}=-\int_{Q}[\nabla \Delta(u \cdot \nabla u)-u \cdot \nabla \nabla \Delta u] \cdot \nabla \Delta u d x
$$

By using the following commutator estimate due to Kato and Ponce (see [16]),

$$
\left\|\nabla^{3}(f g)-f \nabla^{3} g\right\|_{L^{2}} \leq C\left(\|\nabla f\|_{L^{\infty}}\left\|\nabla^{2} g\right\|_{L^{2}}+\left\|\nabla^{3} f\right\|_{L^{2}}\|g\|_{L^{\infty}}\right),
$$

we can estimate $J_{1}$ as follows:

$$
\begin{aligned}
J_{1} & \leq C\|\nabla \Delta(u \cdot \nabla u)-u \cdot \nabla \nabla \Delta u\|_{L^{2}}\|\nabla \Delta u\|_{L^{2}} \\
& \leq C\|\nabla u\|_{L^{\infty}}\|\nabla \Delta u\|_{L^{2}}^{2} .
\end{aligned}
$$

For $J_{2}$, after integration by parts, by (2.5), we obtain

$$
\begin{aligned}
J_{2} & =-\int_{Q} \Delta\left(\frac{\delta E}{\delta \phi} \nabla \phi\right) \Delta^{2} u d x \\
& \leq \frac{\mu}{4}\left\|\Delta^{2} u\right\|_{L^{2}}^{2}+C\left\|\Delta\left(\frac{\delta E}{\delta \phi} \nabla \phi\right)\right\|_{L^{2}}^{2} \\
& \leq \frac{\mu}{4}\left\|\Delta^{2} u\right\|_{L^{2}}^{2}+C\left(\left\|\Delta \frac{\delta E}{\delta \phi} \nabla \phi\right\|_{L^{2}}^{2}+2\left\|\nabla \frac{\delta E}{\delta \phi} \nabla^{2} \phi\right\|_{L^{2}}^{2}+\left\|\frac{\delta E}{\delta \phi} \nabla \Delta \phi\right\|_{L^{2}}^{2}\right) \\
& \leq \frac{\mu}{4}\left\|\Delta^{2} u\right\|_{L^{2}}^{2}+C\left(\left\|\Delta \frac{\delta E}{\delta \phi}\right\|_{L^{2}}^{2}\|\nabla \phi\|_{L^{\infty}}^{2}+\left\|\nabla \frac{\delta E}{\delta \phi}\right\|_{L^{3}}^{2}\left\|\nabla^{2} \phi\right\|_{L^{6}}^{2}+\left\|\frac{\delta E}{\delta \phi}\right\|_{L^{\infty}}^{2}\|\nabla \Delta \phi\|_{L^{2}}^{2}\right) \\
& \leq \frac{\mu}{4}\left\|\Delta^{2} u\right\|_{L^{2}}^{2}+C\left(\left\|\Delta \frac{\delta E}{\delta \phi}\right\|_{L^{2}}^{2}+\left\|\frac{\delta E}{\delta \phi}\right\|_{L^{2}}^{2}+1\right) .
\end{aligned}
$$

Combining (2.22)-(2.24), we deduce that

$$
\frac{d}{d t}\|\nabla \Delta u\|_{L^{2}}^{2}+\frac{3 \mu}{2}\left\|\Delta^{2} u\right\|_{L^{2}}^{2} \leq C\left(\|\nabla u\|_{L^{\infty}}+\left\|\frac{\delta E}{\delta \phi}\right\|_{L^{2}}^{2}+1\right)\left(\|\nabla \Delta u\|_{L^{2}}^{2}+\left\|\Delta \frac{\delta E}{\delta \phi}\right\|_{L^{2}}^{2}+1\right) .
$$


To obtain the desired estimates for $\phi$, we start with

$$
\begin{aligned}
\frac{1}{2} \frac{d}{d t}\left\|\Delta \frac{\delta E}{\delta \phi}\right\|_{L^{2}}^{2}= & \int_{Q} \frac{\partial}{\partial t} \Delta \frac{\delta E}{\delta \phi} \cdot \Delta \frac{\delta E}{\delta \phi} d x \\
= & \int_{Q} \frac{\partial}{\partial t} \Delta\left[k g(\phi)+M_{1}(A(\phi)-\alpha)+M_{2}(B(\phi)-\beta) f(\phi)\right] \cdot \Delta \frac{\delta E}{\delta \phi} d x \\
= & \int_{Q} \frac{\partial}{\partial t} \Delta\left[k g(\phi)+M_{2}(B(\phi)-\beta) f(\phi)\right] \cdot \Delta \frac{\delta E}{\delta \phi} d x \\
= & \int_{Q} \frac{\partial}{\partial t}\left[k g(\phi)+M_{2}(B(\phi)-\beta) f(\phi)\right] \cdot \Delta^{2} \frac{\delta E}{\delta \phi} d x \\
= & -k \int_{Q} \frac{\partial}{\partial t} \Delta f(\phi) \cdot \Delta^{2} \frac{\delta E}{\delta \phi} d x+\frac{k}{\varepsilon^{2}} \int_{Q} \frac{\partial}{\partial t}\left[\left(3 \phi^{2}-1\right) f(\phi)\right] \cdot \Delta^{2} \frac{\delta E}{\delta \phi} d x \\
& +M_{2} \frac{d}{d t} B(\phi) \int_{Q} f(\phi) \cdot \Delta^{2} \frac{\delta E}{\delta \phi} d x+M_{2}(B(\phi)-\beta) \int_{Q} \frac{\partial}{\partial t} f(\phi) \cdot \Delta^{2} \frac{\delta E}{\delta \phi} d x \\
:= & K_{1}+K_{2}+K_{3}+K_{4} .
\end{aligned}
$$

Now we are in a position to estimate the terms $K_{i}(i=1,2,3,4)$ one by one. For $K_{1}$, we split it into the following two parts:

$$
\begin{aligned}
K_{1} & =k \varepsilon \int_{Q} \Delta^{2} \frac{\partial \phi}{\partial t} \cdot \Delta^{2} \frac{\delta E}{\delta \phi} d x-\frac{k}{\varepsilon} \int_{Q} \frac{\partial}{\partial t} \Delta\left(\phi^{3}-\phi\right) \cdot \Delta^{2} \frac{\delta E}{\delta \phi} d x \\
& :=K_{11}+K_{12} .
\end{aligned}
$$

For $K_{11}$, by using Leibniz's rule, we deduce from (1.7) that

$$
\begin{aligned}
K_{11}= & -k \varepsilon \gamma\left\|\Delta^{2} \frac{\delta E}{\delta \phi}\right\|_{L^{2}}^{2}-k \varepsilon \int_{Q} \Delta^{2}(u \cdot \nabla \phi) \cdot \Delta^{2} \frac{\delta E}{\delta \phi} d x \\
\leq & -\frac{15 k \varepsilon \gamma}{16}\left\|\Delta^{2} \frac{\delta E}{\delta \phi}\right\|_{L^{2}}^{2}+C\left\|\Delta^{2}(u \cdot \nabla \phi)\right\|_{L^{2}}^{2} \\
\leq & -\frac{15 k \varepsilon \gamma}{16}\left\|\Delta^{2} \frac{\delta E}{\delta \phi}\right\|_{L^{2}}^{2}+C\left(\left\|\Delta^{2} u \cdot \nabla \phi\right\|_{L^{2}}^{2}+4\|\nabla \Delta u \cdot \nabla \nabla \phi\|_{L^{2}}^{2}\right. \\
& \left.+6\|\Delta u \cdot \nabla \Delta \phi\|_{L^{2}}^{2}+4\|\nabla u \cdot \nabla \nabla \Delta \phi\|_{L^{2}}^{2}+\left\|u \cdot \nabla \Delta^{2} \phi\right\|_{L^{2}}^{2}\right) \\
\leq & -\frac{15 k \varepsilon \gamma}{16}\left\|\Delta^{2} \frac{\delta E}{\delta \phi}\right\|_{L^{2}}^{2}+C\left(\|\nabla \phi\|_{L^{\infty}}^{2}\left\|\Delta^{2} u\right\|_{L^{2}}^{2}+\|\nabla \Delta u\|_{L^{3}}^{2}\left\|\nabla^{2} \phi\right\|_{L^{6}}^{2}\right. \\
& \left.+\|\Delta u\|_{L^{6}}^{2}\|\nabla \Delta \phi\|_{L^{3}}^{2}+\|\nabla u\|_{L^{\infty}}^{2}\left\|\Delta^{2} \phi\right\|_{L^{2}}^{2}+\|u\|_{L^{3}}^{2}\left\|\nabla^{5} \phi\right\|_{L^{6}}^{2}\right) \\
\leq & -\frac{15 k \varepsilon \gamma}{16}\left\|\Delta^{2} \frac{\delta E}{\delta \phi}\right\|_{L^{2}}^{2}+\tilde{C}\left\|\Delta^{2} u\right\|_{L^{2}}^{2}+C\left(\left\|\frac{\delta E}{\delta \phi}\right\|_{L^{2}}^{2}+1\right)\left(\|\nabla \Delta u\|_{L^{2}}^{2}+1\right) \\
& +C\left(\|\nabla u\|_{L^{2}}^{2}+1\right)\left(\left\|\Delta \frac{\delta E}{\delta \phi}\right\|_{L^{2}}^{2}+1\right) \\
\leq & -\frac{15 k \varepsilon \gamma}{16}\left\|\Delta^{2} \frac{\delta E}{\delta \phi}\right\|_{L^{2}}^{2}+\tilde{C}\left\|\Delta^{2} u\right\|_{L^{2}}^{2} \\
& +C\left(\|\nabla u\|_{L^{2}}^{2}+\left\|\frac{\delta E}{\delta \phi}\right\|_{L^{2}}^{2}+1\right)\left(\|\nabla \Delta u\|_{L^{2}}^{2}+\left\|\Delta \frac{\delta E}{\delta \phi}\right\|_{L^{2}}^{2}+1\right)
\end{aligned}
$$


where we have used the facts $\left\|\Delta^{2} \phi\right\|_{L^{2}}^{2} \leq C\left(\left\|\frac{\delta E}{\delta \phi}\right\|_{L^{2}}^{2}+1\right)$ and $\left\|\nabla^{5} \phi\right\|_{L^{6}}^{2} \leq C\left\|\nabla^{6} \phi\right\|_{L^{2}}^{2} \leq C\left(\left\|\Delta \frac{\delta E}{\delta \phi}\right\|_{L^{2}}^{2}+\right.$ 1). We emphasize here that the constant $\tilde{C}$ in (2.28) depending only on $\left\|u_{0}\right\|_{H^{1}},\left\|\phi_{0}\right\|_{H^{3}}, K$ and coefficients of the system due to the estimate (2.5). For $K_{12}$, by using (1.7) again, we obtain

$$
\begin{aligned}
K_{12}= & -\frac{k}{\varepsilon} \int_{Q} \frac{\partial}{\partial t} \Delta\left(\phi^{3}-\phi\right) \cdot \Delta^{2} \frac{\delta E}{\delta \phi} d x=-\frac{6 k}{\varepsilon} \int_{Q} \frac{\partial\left(|\nabla \phi|^{2} \phi\right)}{\partial t} \cdot \Delta^{2} \frac{\delta E}{\delta \phi} d x \\
& -\frac{3 k}{\varepsilon} \int_{Q} \frac{\partial\left(\phi^{2} \Delta \phi\right)}{\partial t} \cdot \Delta^{2} \frac{\delta E}{\delta \phi} d x+\frac{k}{\varepsilon} \int_{Q} \Delta \frac{\partial \phi}{\partial t} \cdot \Delta^{2} \frac{\delta E}{\delta \phi} d x \\
= & -\frac{12 k}{\varepsilon} \int_{Q} \phi \nabla \phi \nabla \frac{\partial \phi}{\partial t} \cdot \Delta^{2} \frac{\delta E}{\delta \phi} d x-\frac{6 k}{\varepsilon} \int_{Q}|\nabla \phi|^{2} \frac{\partial \phi}{\partial t} \cdot \Delta^{2} \frac{\delta E}{\delta \phi} d x \\
& -\frac{6 k}{\varepsilon} \int_{Q} \phi \Delta \phi \frac{\partial \phi}{\partial t} \cdot \Delta^{2} \frac{\delta E}{\delta \phi} d x-\frac{3 k}{\varepsilon} \int_{Q} \phi^{2} \Delta \frac{\partial \phi}{\partial t} \cdot \Delta^{2} \frac{\delta E}{\delta \phi} d x \\
& +\frac{k}{\varepsilon} \int_{Q} \Delta \frac{\partial \phi}{\partial t} \cdot \Delta^{2} \frac{\delta E}{\delta \phi} d x:=\sum_{i=1}^{5} K_{12 i} .
\end{aligned}
$$

Note that, by using (2.5), we can easily deduce from (1.7) that

$$
\begin{gathered}
\left\|\frac{\partial \phi}{\partial t}\right\|_{L^{2}} \leq C\left(\|u \cdot \nabla \phi\|_{L^{2}}+\left\|\frac{\delta E}{\delta \phi}\right\|_{L^{2}}\right) \leq C\left(\left\|\frac{\delta E}{\delta \phi}\right\|_{L^{2}}+1\right), \\
\left\|\nabla \frac{\partial \phi}{\partial t}\right\|_{L^{2}} \leq C\left(\|\nabla u \cdot \nabla \phi\|_{L^{2}}+\left\|u \cdot \nabla^{2} \phi\right\|_{L^{2}}+\left\|\nabla \frac{\delta E}{\delta \phi}\right\|_{L^{2}}\right) \\
\leq C\left(\|\nabla u\|_{L^{2}}\|\nabla \phi\|_{L^{\infty}}+\|u\|_{L^{3}}\left\|\nabla^{2} \phi\right\|_{L^{6}}+\left\|\nabla \frac{\delta E}{\delta \phi}\right\|_{L^{2}}\right) \\
\leq C\left(\|\nabla u\|_{L^{2}}+\left\|\frac{\delta E}{\delta \phi}\right\|_{L^{2}}+\left\|\Delta \frac{\delta E}{\delta \phi}\right\|_{L^{2}}+1\right), \\
\left\|\Delta \frac{\partial \phi}{\partial t}\right\|_{L^{2}} \leq C\left(\|\Delta u \cdot \nabla \phi\|_{L^{2}}+\left\|\nabla u \cdot \nabla^{2} \phi\right\|_{L^{2}}+\|u \cdot \nabla \Delta \phi\|_{L^{2}}+\left\|\Delta \frac{\delta E}{\delta \phi}\right\|_{L^{2}}\right) \\
\leq C\left(\|\Delta u\|_{L^{6}}\|\nabla \phi\|_{L^{3}}+\|\nabla u\|_{L^{3}}\left\|\nabla^{2} \phi\right\|_{L^{6}}+\|u\|_{L^{\infty}}\|\nabla \Delta \phi\|_{L^{2}}+\left\|\Delta \frac{\delta E}{\delta \phi}\right\|_{L^{2}}\right) \\
\leq C\left(\|\nabla \Delta u\|_{L^{2}}+\left\|\Delta \frac{\delta E}{\delta \phi}\right\|_{L^{2}}+1\right), \\
\left\|\frac{\partial f(\phi)}{\partial t}\right\|_{L^{2}} \leq C\left(\left\|\Delta \frac{\partial \phi}{\partial t}\right\|_{L^{2}}+\left\|\frac{\partial}{\partial t}\left(\phi^{3}-\phi\right)\right\|_{L^{2}}\right) \\
\leq C\left(\left\|\Delta \frac{\partial \phi}{\partial t}\right\|_{L^{2}}+3\|\phi\|_{L^{\infty}}^{2}\left\|\frac{\partial \phi}{\partial t}\right\|_{L^{2}}+\left\|\frac{\partial \phi}{\partial t}\right\|_{L^{2}}\right) \\
\leq C\left(\|\nabla \Delta u\|_{L^{2}}+\left\|\Delta \frac{\delta E}{\delta \phi}\right\|_{L^{2}}+\left\|\frac{\delta E}{\delta \phi}\right\|_{L^{2}}+1\right) .
\end{gathered}
$$

Hence, we can estimate the terms $K_{12 i}(i=1,2,3,4,5)$ as follows:

$$
K_{121} \leq \frac{k \varepsilon \gamma}{16}\left\|\Delta^{2} \frac{\delta E}{\delta \phi}\right\|_{L^{2}}^{2}+C\left\|\phi \nabla \phi \nabla \frac{\partial \phi}{\partial t}\right\|_{L^{2}}^{2}
$$




$$
\begin{aligned}
& \leq \frac{k \varepsilon \gamma}{16}\left\|\Delta^{2} \frac{\delta E}{\delta \phi}\right\|_{L^{2}}^{2}+C\|\phi\|_{L^{\infty}}^{2}\|\nabla \phi\|_{L^{\infty}}^{2}\left\|\nabla \frac{\partial \phi}{\partial t}\right\|_{L^{2}}^{2} \\
& \leq \frac{k \varepsilon \gamma}{16}\left\|\Delta^{2} \frac{\delta E}{\delta \phi}\right\|_{L^{2}}^{2}+C\left(\|\nabla u\|_{L^{2}}^{2}+\left\|\Delta \frac{\delta E}{\delta \phi}\right\|_{L^{2}}^{2}+\left\|\frac{\delta E}{\delta \phi}\right\|_{L^{2}}^{2}\right) ; \\
& K_{122} \leq \frac{k \varepsilon \gamma}{16}\left\|\Delta^{2} \frac{\delta E}{\delta \phi}\right\|_{L^{2}}^{2}+C\|\nabla \phi\|_{L^{\infty}}^{4}\left\|\frac{\partial \phi}{\partial t}\right\|_{L^{2}}^{2} \\
& \leq \frac{k \varepsilon \gamma}{16}\left\|\Delta^{2} \frac{\delta E}{\delta \phi}\right\|_{L^{2}}^{2}+C\left(\left\|\frac{\delta E}{\delta \phi}\right\|_{L^{2}}^{2}+1\right) ; \\
& K_{123} \leq \frac{k \varepsilon \gamma}{16}\left\|\Delta^{2} \frac{\delta E}{\delta \phi}\right\|_{L^{2}}^{2}+C\|\phi\|_{L^{\infty}}^{2}\left\|\frac{\partial \phi}{\partial t}\right\|_{L^{3}}^{2}\|\Delta \phi\|_{L^{6}}^{2} \\
& \leq \frac{k \varepsilon \gamma}{16}\left\|\Delta^{2} \frac{\delta E}{\delta \phi}\right\|_{L^{2}}^{2}+C\left(\|u \cdot \nabla \phi\|_{L^{3}}^{2}+\left\|\frac{\delta E}{\delta \phi}\right\|_{L^{3}}^{2}\right) \\
& \leq \frac{k \varepsilon \gamma}{16}\left\|\Delta^{2} \frac{\delta E}{\delta \phi}\right\|_{L^{2}}^{2}+C\left(\|u\|_{L^{6}}^{2}\|\nabla \phi\|_{L^{6}}^{2}+\left\|\Delta \frac{\delta E}{\delta \phi}\right\|_{L^{2}}^{2}+\left\|\frac{\delta E}{\delta \phi}\right\|_{L^{2}}^{2}\right) \\
& \leq \frac{k \varepsilon \gamma}{16}\left\|\Delta^{2} \frac{\delta E}{\delta \phi}\right\|_{L^{2}}^{2}+C\left(\|\nabla u\|_{L^{2}}^{2}+\left\|\Delta \frac{\delta E}{\delta \phi}\right\|_{L^{2}}^{2}+\left\|\frac{\delta E}{\delta \phi}\right\|_{L^{2}}^{2}\right) ; \\
& K_{124} \leq \frac{k \varepsilon \gamma}{16}\left\|\Delta^{2} \frac{\delta E}{\delta \phi}\right\|_{L^{2}}^{2}+C\|\phi\|_{L^{\infty}}^{2}\left\|\Delta \frac{\partial \phi}{\partial t}\right\|_{L^{2}}^{2} \\
& \leq \frac{k \varepsilon \gamma}{16}\left\|\Delta^{2} \frac{\delta E}{\delta \phi}\right\|_{L^{2}}^{2}+C\left(\|\nabla \Delta u\|_{L^{2}}^{2}+\left\|\Delta \frac{\delta E}{\delta \phi}\right\|_{L^{2}}^{2}+1\right) ; \\
& K_{125} \leq \frac{k \varepsilon \gamma}{16}\left\|\Delta^{2} \frac{\delta E}{\delta \phi}\right\|_{L^{2}}^{2}+C\left\|\Delta \frac{\partial \phi}{\partial t}\right\|_{L^{2}}^{2} \\
& \leq \frac{k \varepsilon \gamma}{16}\left\|\Delta^{2} \frac{\delta E}{\delta \phi}\right\|_{L^{2}}^{2}+C\left(\|\nabla \Delta u\|_{L^{2}}^{2}+\left\|\Delta \frac{\delta E}{\delta \phi}\right\|_{L^{2}}^{2}+1\right) .
\end{aligned}
$$

Putting estimates (2.30)-(2.34) together, we obtain

$$
K_{12} \leq \frac{5 k \varepsilon \gamma}{16}\left\|\Delta^{2} \frac{\delta E}{\delta \phi}\right\|_{L^{2}}^{2}+C\left(\|\nabla \Delta u\|_{L^{2}}^{2}+\left\|\Delta \frac{\delta E}{\delta \phi}\right\|_{L^{2}}^{2}+\left\|\frac{\delta E}{\delta \phi}\right\|_{L^{2}}^{2}+1\right) .
$$

Taking (2.28) and (2.35) into (2.27), we get

$$
\begin{aligned}
K_{1} \leq & -\frac{5 k \varepsilon \gamma}{8}\left\|\Delta^{2} \frac{\delta E}{\delta \phi}\right\|_{L^{2}}^{2}+\tilde{C}\left\|\Delta^{2} u\right\|_{L^{2}}^{2} \\
& +C\left(\|\nabla u\|_{L^{2}}^{2}+\left\|\frac{\delta E}{\delta \phi}\right\|_{L^{2}}^{2}+1\right)\left(\|\nabla \Delta u\|_{L^{2}}^{2}+\left\|\Delta \frac{\delta E}{\delta \phi}\right\|_{L^{2}}^{2}+1\right) .
\end{aligned}
$$

For $K_{2}$,

$$
\begin{aligned}
K_{2} & =\frac{6 k}{\varepsilon^{2}} \int_{Q} \phi f(\phi) \frac{\partial \phi}{\partial t} \cdot \Delta^{2} \frac{\delta E}{\delta \phi} d x+\frac{k}{\varepsilon^{2}} \int_{Q}\left(3 \phi^{2}-1\right) \frac{\partial f(\phi)}{\partial t} \cdot \Delta^{2} \frac{\delta E}{\delta \phi} d x \\
& =-\frac{6 k}{\varepsilon} \int_{Q} \phi \Delta \phi \frac{\partial \phi}{\partial t} \cdot \Delta^{2} \frac{\delta E}{\delta \phi} d x+\frac{6 k}{\varepsilon^{3}} \int_{Q} \phi^{2}\left(\phi^{2}-1\right) \frac{\partial \phi}{\partial t} \cdot \Delta^{2} \frac{\delta E}{\delta \phi} d x
\end{aligned}
$$




$$
\begin{aligned}
& +\frac{k}{\varepsilon^{2}} \int_{Q}\left(3 \phi^{2}-1\right) \frac{\partial f(\phi)}{\partial t} \cdot \Delta^{2} \frac{\delta E}{\delta \phi} d x \\
\leq & \frac{k \varepsilon \gamma}{8}\left\|\Delta^{2} \frac{\delta E}{\delta \phi}\right\|_{L^{2}}^{2}+C\left(\|\phi\|_{L^{\infty}}^{2}\|\Delta \phi\|_{L^{6}}^{2}\left\|\frac{\partial \phi}{\partial t}\right\|_{L^{3}}^{2}+\|\phi\|_{L^{\infty}}^{4}\left\|\phi^{2}-1\right\|_{L^{\infty}}^{2}\left\|\frac{\partial \phi}{\partial t}\right\|_{L^{2}}^{2}\right. \\
& \left.+\left\|3 \phi^{2}-1\right\|_{L^{\infty}}^{2}\left\|\frac{\partial f(\phi)}{\partial t}\right\|_{L^{2}}^{2}\right) \\
\leq & \frac{k \varepsilon \gamma}{8}\left\|\Delta^{2} \frac{\delta E}{\delta \phi}\right\|_{L^{2}}^{2}+C\left(\left\|\frac{\partial \phi}{\partial t}\right\|_{L^{3}}^{2}+\left\|\frac{\partial \phi}{\partial t}\right\|_{L^{2}}^{2}+\left\|\frac{\partial f(\phi)}{\partial t}\right\|_{L^{2}}^{2}\right) \\
\leq & \frac{k \varepsilon \gamma}{8}\left\|\Delta^{2} \frac{\delta E}{\delta \phi}\right\|_{L^{2}}^{2}+C\left(\|\nabla \Delta u\|_{L^{2}}^{2}+\left\|\Delta \frac{\delta E}{\delta \phi}\right\|_{L^{2}}^{2}+\left\|\frac{\delta E}{\delta \phi}\right\|_{L^{2}}^{2}+1\right) .
\end{aligned}
$$

For $K_{3}$

$$
\begin{aligned}
K_{3} & \leq C\left|\frac{d B(\phi)}{d t}\right|\|f(\phi)\|_{L^{2}}\left\|\Delta^{2} \frac{\delta E}{\delta \phi}\right\|_{L^{2}} \\
& \leq \frac{k \varepsilon \gamma}{8}\left\|\Delta^{2} \frac{\delta E}{\delta \phi}\right\|_{L^{2}}^{2}+C\left(\|\nabla \phi\|_{L^{2}}\left\|\nabla \frac{\partial \phi}{\partial t}\right\|_{L^{2}}+\left\|\phi^{3}-\phi\right\|_{L^{2}}\left\|\frac{\partial \phi}{\partial t}\right\|_{L^{2}}\right)^{2}\|f(\phi)\|_{L^{2}}^{2} \\
& \leq \frac{k \varepsilon \gamma}{8}\left\|\Delta^{2} \frac{\delta E}{\delta \phi}\right\|_{L^{2}}^{2}+C\left(\|\nabla u\|_{L^{2}}^{2}+\left\|\Delta \frac{\delta E}{\delta \phi}\right\|_{L^{2}}^{2}+\left\|\frac{\delta E}{\delta \phi}\right\|_{L^{2}}^{2}+1\right) .
\end{aligned}
$$

For $K_{4}$,

$$
\begin{aligned}
K_{4} & \leq C|B(\phi)-\beta|\left\|\frac{\partial f(\phi)}{\partial t}\right\|_{L^{2}}\left\|\Delta^{2} \frac{\delta E}{\delta \phi}\right\|_{L^{2}} \\
& \leq \frac{k \varepsilon \gamma}{8}\left\|\Delta^{2} \frac{\delta E}{\delta \phi}\right\|_{L^{2}}^{2}+C\left(\|\nabla \phi\|_{L^{2}}^{2}+\left\|\phi^{2}-1\right\|_{L^{2}}^{2}\right)^{2}\left\|\frac{\partial f(\phi)}{\partial t}\right\|_{L^{2}}^{2} \\
& \leq \frac{k \varepsilon \gamma}{8}\left\|\Delta^{2} \frac{\delta E}{\delta \phi}\right\|_{L^{2}}^{2}+C\left(\|\nabla \Delta u\|_{L^{2}}^{2}+\left\|\Delta \frac{\delta E}{\delta \phi}\right\|_{L^{2}}^{2}+\left\|\frac{\delta E}{\delta \phi}\right\|_{L^{2}}^{2}+1\right) .
\end{aligned}
$$

Taking (2.36)-(2.39) into (2.26), we conclude that

$$
\begin{aligned}
\frac{d}{d t}\left\|\Delta \frac{\delta E}{\delta \phi}\right\|_{L^{2}}^{2}+ & \frac{k \varepsilon \gamma}{2}\left\|\Delta^{2} \frac{\delta E}{\delta \phi}\right\|_{L^{2}}^{2} \leq \tilde{C}\left\|\Delta^{2} u\right\|_{L^{2}}^{2} \\
& +C\left(\|\nabla u\|_{L^{2}}^{2}+\left\|\frac{\delta E}{\delta \phi}\right\|_{L^{2}}^{2}+1\right)\left(\|\nabla \Delta u\|_{L^{2}}^{2}+\left\|\Delta \frac{\delta E}{\delta \phi}\right\|_{L^{2}}^{2}+1\right) .
\end{aligned}
$$

Set

$$
\eta=\frac{\mu}{2 \tilde{C}}
$$

Multiplying $\eta$ by (2.40), adding (2.25) together, we obtain

$$
\begin{aligned}
\frac{d}{d t}\left(\|\nabla \Delta u\|_{L^{2}}^{2}\right. & \left.+\eta\left\|\Delta \frac{\delta E}{\delta \phi}\right\|_{L^{2}}^{2}\right)+\mu\left\|\Delta^{2} u\right\|_{L^{2}}^{2}+\frac{k \varepsilon \gamma \eta}{2}\left\|\Delta^{2} \frac{\delta E}{\delta \phi}\right\|_{L^{2}}^{2} \\
& \leq C\left(\|\nabla u\|_{L^{\infty}}+\|\nabla u\|_{L^{2}}^{2}+\left\|\frac{\delta E}{\delta \phi}\right\|_{L^{2}}^{2}+1\right)\left(\|\nabla \Delta u\|_{L^{2}}^{2}+\eta\left\|\Delta \frac{\delta E}{\delta \phi}\right\|_{L^{2}}^{2}+e\right),
\end{aligned}
$$

where the constant $C$ may depend on $\eta$. Putting the inequality (2.41) and the basic energy inequality (1.12) together imply that

$$
\frac{d}{d t}\left(\|u\|_{H^{3}}^{2}+\eta\left\|\frac{\delta E}{\delta \phi}\right\|_{H^{2}}^{2}\right) \leq C\left(\|\nabla u\|_{L^{\infty}}+\|\nabla u\|_{L^{2}}^{2}+\left\|\frac{\delta E}{\delta \phi}\right\|_{L^{2}}^{2}+1\right)\left(\|u\|_{H^{3}}^{2}+\eta\left\|\frac{\delta E}{\delta \phi}\right\|_{H^{2}}^{2}+e\right) .
$$


Finally, we end the proof of Theorem 1.1 by applying Gronwall's inequality. Set

$$
m(t)=e+\|u\|_{H^{3}}^{2}+\eta\left\|\frac{\delta E}{\delta \phi}\right\|_{H^{2}}^{2} .
$$

Then by (2.42), we see that

$$
\frac{d m(t)}{d t} \leq C\left(\|\nabla u\|_{L^{\infty}}+\|\nabla u\|_{L^{2}}^{2}+\left\|\frac{\delta E}{\delta \phi}\right\|_{L^{2}}^{2}+1\right) m(t) .
$$

By combining the following logarithmic Sobolev inequality (see [2]):

$$
\|\nabla u\|_{L^{\infty}} \leq C\left(1+\|\omega\|_{L^{2}}+\|\omega\|_{L^{\infty}} \ln \left(e+\|u\|_{H^{3}}\right)\right)
$$

and (2.5), we get

$$
\|\nabla u\|_{L^{\infty}} \leq C\left(1+\|\omega\|_{L^{\infty}} \ln \left(e+\|u\|_{H^{3}}\right)\right) .
$$

This estimate with (2.43) and the inequality $\ln m(t) \geq 1$ yield that

$$
\frac{d}{d t} \ln m(t) \leq C\left(\|\omega\|_{L^{\infty}}+\|\nabla u\|_{L^{2}}^{2}+\left\|\frac{\delta E}{\delta \phi}\right\|_{L^{2}}^{2}+1\right) \ln m(t) .
$$

By applying Gronwall's inequality, we obtain

$$
m(t) \leq \exp \left\{\ln m(0) \exp \left(C(1+t)+C \int_{0}^{t}\|\omega(s)\|_{L^{\infty}} d s\right)\right\}
$$

for any $0<t \leq T$, where $m(0)$ is a constant depending only on $\left\|u_{0}\right\|_{H^{3}},\left\|\phi_{0}\right\|_{H^{6}}$ and coefficients of the system. Note that

$$
\|\phi\|_{H^{6}}^{2} \leq C\left(\left\|\Delta \frac{\delta E}{\delta \phi}\right\|_{L^{2}}^{2}+1\right)
$$

This fact with (2.46) imply immediately that

$$
\sup _{0 \leq t \leq T}\left(\|u(\cdot, t)\|_{H^{3}}+\|\phi(\cdot, t)\|_{H^{6}}\right) \leq C .
$$

We complete the proof of Theorem 1.1

\section{References}

[1] M. Abkarian, C. Lartigue, A. Viallat, Tank treading and unbinding of deformable vesicles in shear flow: Determination of the lift force, Phys. Rev. Lett. 88 (2002), 068103.

[2] J. T. Beale, T. Kato, A. Majda, Remarks on breakdown of smooth solutions for the 3D Euler equations, Commun. Math. Phys. 94 (1984), 61-66.

[3] H. Beirão da Veiga, A new regularity class for the Navier-Stokes equations in $\mathbb{R}^{n}$, Chin. Ann. Math. Ser. B: 16 (1995), 407-412. 
[4] R. S. Chadwick, Axisymmetric indentation of a thin incompressible elastic layer, SIAM J. Appl. Math. 62(5) (2002), 1520-1530.

[5] K. H. de Haas, C. Blom, D. van den Ende, M. H. G. Duits, J. Mellema, Deformation of giant lipid bilayer vesicles in shear flow, Phys. Rev. E 56 (1997), 7132-7137.

[6] Q. Du, M. Li, C. Liu, Analysis of a phase field Navier-Stokes vesicle-fluid interaction model, Discrete Contin. Dyn. Syst. B 8(3) (2007), 539-556.

[7] Q. Du, C. Liu, R. Ryham, X. Wang, A phase field formulation of the Willmore problem, Nonlinearity 18 (2005), 1249-1267.

[8] Q. Du, C. Liu, R. Ryham, X. Wang, Phase field modeling of the spontaneous curvature effect in cell membranes, Commun. Pure Appl. Anal. 4 (2005), 537-548.

[9] Q. Du, C. Liu, R. Ryham, X. Wang, Energetic variational approaches in modeling vesicle and fluid interactions, Physica D 238 (2009), 923-930.

[10] Q. Du, C. Liu, X. Wang, A phase field approach in the numerical study of the elastic bending energy for vesicle membranes, J. Computational Physics 198 (2004), 450-468.

[11] Q. Du, C. Liu, X. Wang, Retrieving topological information for phase field models, SIAM J. Appl. Math. 65 (2005), 1913-1932.

[12] Q. Du, C. Liu, X. Wang, Simulating the deformation of vesicle membranes under elastic bending energy in three dimensions, J. Computational Physics 212 (2006), 757-777.

[13] W. Helfrich, Elastic properties of lipid bilayers: theory and possible experiments, Z. Naturforsch. 28 c (1973), 693-703.

[14] Y. Giga, Solutions for semilinear parabolic equations in $L^{p}$ and regularity of weak solutions of the Navier-Stokes system, J. Differential Equations 61 (1986), 186-212.

[15] V. Kantsler, V. Steinberg, Orientation and dynamics of a vesicle in tank-treading motion in shear flow, Phys. Rev. Lett. 95 (2005), 258101.

[16] T. Kato, G. Ponce, Commutator estimates and the Euler and Navier-Stokes equations, Comm. Pure Appl. Math. 41 (1988), 891-907.

[17] H. Kozono, T. Ogawa, Y. Taniuchi, The critical Sobolev inequalities in Besov spaces and regularity criterion to some semi-linear evolution equations, Math. Z. 242 (2002), 251-278.

[18] H. Kozono, Y. Taniuchi, Bilinear estimates in BMO and the Navier-Stokes equations, Math. Z. 235 (2000), 173-194.

[19] Y. Liu, T. Takahashi, M. Tucsnak, Strong solution for a phase field Navier-Stokes vesicle fluid interaction model, J. Math. Fluid Mech. 14 (2011), 177-195. 
[20] L. Miao, U. Seifert, M. Wortis, H.-G Döbereiner, Budding transitions of fluid-bilayer vesicle: The effect of area-difference elasticity, Phys. Rev. E 49 (1994), 5389-5407.

[21] O. Mouritsen, Life-As a Matter of Fat: The Emerging Science of Lipidomics, Springer, Berlin, 2005.

[22] Z. Ou-Yang, W. Helfrich, Bending energy of vesicle membranes: General expressions for the first, second and third variation of the shape energy and applications to spheres and cylinders, Phys. Rev. A 39 (1989), 5280-5288.

[23] G. Prodi, Un teorema di unicità per le equazioni di Navier-Stokes, Ann. Math. Pura Appl. 48(1) (1959), 173-182.

[24] U. Seifert, Curvature-induced lateral phase segregation in two-component vesicles, Phys. Rev. Lett. 70 (1993), 1335-1338.

[25] U. Seifert, R. Lipowsky, Morphology of Vesicles, in: Handbook of Biological Physics, vol. 1, 1995.

[26] J. Serrin, On the interior regularity of weak solutions of the Navier-Stokes equations, Arch. Rational Mech. Anal. 9 (1962), 187-195.

[27] X. Wang, Q. Du, Modelling and simulations of multi-component liquid membranes and open membranes via diffuse interface approaches, J. Math. Biol. 56(3) (2008), 347-371.

[28] H. Wu, X. Xu, Global regularity and stability of a hydrodynamic system modeling vesicle and fluid interactions, arXiv:1202.4869v1. 